\title{
Using behavioral insights to design implementation strategies in public mental health settings: a qualitative study of clinical decision-making
}

Briana S. Last ${ }^{1 *}$ (D), Simone H. Schriger ${ }^{1}$, Carter E. Timon², Hannah E. Frank ${ }^{3,4}$, Alison M. Buttenheim, 5,7, Brittany N. Rudd ${ }^{7,8,9}$, Sara Fernandez-Marcote ${ }^{10}$, Carrie Comeau ${ }^{11}$, Sosunmolu Shoyinka ${ }^{11}$ and Rinad S. Beidas $5^{6,7,8,12,13}$

\begin{abstract}
Background: Trauma-focused cognitive behavioral therapy (TF-CBT) is an evidence-based intervention for youth with posttraumatic stress disorder. An important component of TF-CBT is the trauma narrative (TN), a phase in the intervention in which youth are guided to process the memories, thoughts, and feelings associated with their traumatic experience(s). Previous work has shown that TF-CBT clinicians complete TNs with only half of their clients, yet little is known about what determines TF-CBT clinicians' use of TNs. The behavioral insights literature-an interdisciplinary field studying judgment and decision-making-offers theoretical and empirical tools to conceptualize what drives complex human behaviors and decisions. Drawing from the behavioral insights literature, the present study seeks to understand what determines clinician use of TNs and to generate strategies that target these determinants.
\end{abstract}

Methods: Through semi-structured qualitative interviews, we sought the perspectives of trained TF-CBT clinicians working in public mental health settings across the city of Philadelphia $(N=17)$ to understand their decisions to use TNs with clients. We analyzed the qualitative data using a coding approach informed by the behavioral insights literature. We used an iterative process of structured hypothesis generation, aided by a behavioral insights guide, and rapid validation informed by behavioral insights to uncover the determinants of TN use. We then generated implementation strategies that targeted these determinants using the "Easy Attractive Social Timely" framework, a behavioral insights design approach.

Results: We generated and validated three broad themes about what determines clinician implementation of TNs: decision complexity, clinician affective experience, and agency norms. We hypothesized behavioral insights that underlie these implementation determinants and designed a list of nine corresponding behavioral insights strategies that may facilitate TN implementation.

(Continued on next page)

\footnotetext{
* Correspondence: brishiri@sas.upenn.edu

'Department of Psychology, University of Pennsylvania, Philadelphia, PA, USA

Full list of author information is available at the end of the article
} 
(Continued from previous page)

Conclusions: Our study investigated why an effective component of an evidence-based intervention is difficult to implement. We leveraged robust scientific theories and empirical regularities from the behavioral insights literature to understand clinician perspectives on TN implementation. These factors were theoretically linked to implementation strategies. Our work revealed the potential for using behavioral insights in the diagnosis of evidence-based intervention determinants and the design of implementation strategies.

Keywords: Trauma-focused cognitive behavioral therapy (TF-CBT), Behavioral insights, Behavioral economics, Posttraumatic stress disorder, Implementation science, Implementation strategies

\section{Contributions to the literature}

- This study identifies several determinants for why clinicians in public mental health settings complete the trauma narrative, a core component of trauma-focused cognitive behavioral therapy (TF-CBT), with only half of their clients.

- This work is unique in its integration of clinicians' perspectives with the behavioral insights literature.

- This study illustrates a process for how to use behavioral insights - an interdisciplinary corpus of scientific theories and empirical findings - to understand clinicians' decisionmaking and to design implementation strategies.

- These findings contribute to the work of TF-CBT implementation by unveiling the various ways clinicians in public, resourcescarce mental health settings face decision-making challenges.

\section{Background}

There are numerous challenges to the implementation of mental health evidence-based interventions (EBIs; interventions supported by scientific evidence). These challenges are often specific to the structural and organizational factors that constitute the ecosystem of mental health service delivery, the individual decisionmakers involved in implementation, and the client population [1-9]. Though these challenges are generalizable to the implementation of most mental health EBIs, there are also unique challenges to the implementation of specific mental health EBIs that require attention in the development and selection of implementation strategies.

First, mental health EBIs are complex and multicomponent. Most mental health interventions are designed and tested in efficacy trials as complete packages or manualized protocols, yet a limited set of core techniques and principles in mental health EBIs are responsible for their effectiveness [10-12]. With implementation in mind, there is growing recognition by researchers that it is essential to understand which EBI components account for therapeutic change $[13,14]$. Once identified, implementation researchers can prioritize the specific components that have garnered the strongest evidence to target impediments to implementation and to design strategies to overcome these barriers. It is particularly important to target these components because these core techniques are often the most likely to be underused by clinicians [15-18].

Second, it is not always clear how to generate implementation strategies based on stakeholders' first-person perspectives, even with the use of scientific frameworks. Implementation science has long recognized that qualitative research is essential to provide a textured understanding of clinicians' experiences of EBI use [19, 20]. Thus, methods to design and select implementation strategies rely on stakeholder's self-reported barriers and facilitators [21]. One challenge for EBI implementation is to reconcile the growing literature that shows that clinicians, like all humans, lack complete insight into their motivations and behaviors and, further, that their selfreports are conditioned by their organizational and broader social contexts [22]. One potential reconciliation is to apply scientific theories on judgment and decisionmaking to the analysis of stakeholder perspectives. That is, qualitative data can be leveraged to go beyond literal interpretation of clinician self-report-these data can be interpreted using scientific theories of the implicit processes that underlie judgment and decision-making to generate falsifiable causal hypotheses for why EBIs are challenging to implement and why strategies do or do not work.

\section{Behavioral insights}

"Behavioral insights" (an umbrella term referring to discoveries from behavioral economics, cognitive science, and social psychology) can offer scientists tools to address the challenges of implementing complex EBIs [23]. Behavioral insights comprise a set of theoretical principles, frameworks, empirical regularities, and strategies derived from a decades-long, multidisciplinary effort to understand human judgment and decision-making [24]. These insights reveal the ways in which individuals make decisions-individuals tend to have incomplete information, work with enormous constraints on their time and resources, and employ heuristics, or mental shortcuts, to make decisions [25]. Behavioral insights demonstrate how people's computational limits and motivated reasoning shape judgment and decision-making. Importantly, people 
are largely unaware of the biases and mental shortcuts they employ to make decisions [26]. This has implications for the interpretation of self-report data. If people are unaware of their motivations, judgments, and decisions, then selfreport data on EBI implementation may benefit from interpretation using behavioral insights.

In addition to elucidating human judgment and decision-making, the multidisciplinary field of behavioral insights has generated strategies to improve decisionmaking. Rather than attempting to change the ways in which people are systematically biased, behavioral insights strategies leverage these systematic biases to optimize decision-making. A subset of these strategies, known as nudges, alter the "choice architecture," or the way options are presented, to lead decision-makers to behave in predictable ways [27]. Nudges shape choice architectures to influence discrete, one-time decisions. For example, people overwhelmingly tend to choose the status quo or default option [28]. Nudges that make the optimal or more evidence-based decision the default have been effective across a host of domains including dietary choices, medical decisions, financial savings, and education [29-31]. Behavioral insights also encompass strategies that, unlike nudges, require sustained effort. For example, psychologists and implementation scientists have long-recognized that social motivation, incentives, and rewards are crucial levers of behavior change [32-37]. Studies from across the globe suggest that providing women with continuous social support during childbirth using a labor companion encourages them to be mobile, per guidelines, and improves health outcomes for women and babies [38]. Internationally, health service researchers and public health organizations are beginning to study behavioral insights to improve healthcare [39-48], and a new review calls for their integration into implementation science [49].

\section{Study context}

This study was conducted in the city of Philadelphia, where the majority of treatment-seeking youth (55-80\%) in the city receive public mental health services [50,51]. Public mental health services, funded by Medicaid, are administered by the Department of Behavioral Health and Intellectual Disability Services (DBHIDS). Due to the high incidence of trauma exposure in Philadelphia, DBHIDS initiated a full-scale effort to develop a traumainformed behavioral health system in 2011. In 2012, DBHIDS was awarded a National Child Traumatic Stress Initiative Community Treatment and Service Center grant (Category III) from the Substance Abuse and Mental Health Services Administration (SAMHSA) to form the Philadelphia Alliance for Child Trauma Services (PACTS). These grants support building the enduring infrastructure necessary to facilitate implementation rather than focusing on increasing the uptake of particular interventions [1]. PACTS represents a publicacademic partnership that includes policy-makers, public mental health agency leadership, and university-based researchers who have worked collaboratively for the past decade to create a network of trauma-informed care in Philadelphia. In addition to increasing trauma screening and assessment and developing a robust crisis response service, PACTS has supported the training of clinicians in evidence-based trauma treatments.

Of these treatments, trauma-focused cognitive behavioral therapy (TF-CBT) has been a focus [50]. Over twenty randomized controlled trials (RCTs) show that TF-CBT is effective for youth with posttraumatic stress disorder [52-54]. Despite its research base, TF-CBT is not regularly implemented in public mental health settings [55]. Since 2012, ten cohorts of clinicians have been trained in TF-CBT across outpatient public mental health and residential treatment agencies through the PACTS initiative. Training includes 2 days of didactics followed by ongoing consultation provided via bi-weekly consultation calls for eight months with a TF-CBT certified master trainer. Throughout the year, PACTS-trained clinicians are offered to participate in "booster sessions" to fine-tune skills and seek clinical guidance. See [50] for more details on PACTS and TF-CBT training.

Dismantling research demonstrates that TF-CBT is more effective when the trauma narrative $(\mathrm{TN})$ is used [56]. In the $\mathrm{TN}$ phase of treatment, the clinician guides the youth in sharing their memories, thoughts, and feelings related to the traumatic event. The narrative serves several purposes, including systematically desensitizing the child to traumatic memories as well as facilitating emotional processing of the memories to provide the child with a sense of mastery over their traumatic experiences. TF-CBT national trainers (i.e., expert TF-CBT clinicians who have trained over 5000 TF-CBT providers) have identified $\mathrm{TN}$ implementation as the most significant challenge to TF-CBT fidelity [17]. They speculated that clinician discomfort with a directive approach, fear of causing harm, and limited therapeutic skills beyond TF-CBT were significant barriers to TN implementation. To our knowledge, studies of clinicians' perspectives of implementing TNs have not yet been published. However, TF-CBT clinicians in Philadelphia report completing TNs with only half of their clients [50].

\section{Objective}

The present work examines perspectives from clinicians participating in city-wide implementation efforts in Philadelphia to (1) understand the implementation of an active yet underused component (the TN) of an effective and complex psychological EBI (TF-CBT) and (2) use principles from the behavioral insights literature to 
theoretically link this understanding to the development of implementation strategies.

\section{Methods}

In 2018, we conducted qualitative interviews with PACTS clinicians across Philadelphia and asked about their decision-making processes implementing TNs. We adapted a behavioral insights approach to systematically stage the analysis-Narrow, Understand, Discover, Generate, and Evaluate (NUDGE) - and coded the interview data using a guide from the behavioral insights literature-the Behavioral Economics Guide-to arrive at behaviorally informed hypotheses about the determinants of clinicians' TN use $[57,58]$. We leveraged these hypotheses to generate implementation strategies using the behavioral insights-informed Easy Attractive Social and Timely (EAST) framework, which organizes strategies (both nudge and non-nudge) for researchers and policy makers [59].

\section{Participants and study procedure}

Participants were clinicians who had completed training in TF-CBT through PACTS. Clinicians were contacted (either by e-mail or at a "booster" training session) in the spring of 2018 and asked to complete a 10-15-min survey about their perceptions of and past use of TNs. See [60] for more information about the initial survey clinicians completed. Of the 65 clinicians that completed the survey, a subset $(n=26)$ was selected for in-depth qualitative interviews using purposive sampling. Participants who completed qualitative interviews were sampled to capture variability in clinician TN use. On the survey, participants indicated the percentage of TF-CBT clients with whom they used TNs in the past 6 months, whether they intended to use TNs with their TF-CBT clients in the next six months, and how likely it was that they would use TNs with their TF-CBT clients in the next 6 months. Based on these responses, clinicians fell into three groups and were purposely sampled for qualitative interviews from each group, including (1) clinicians with high intentions and high likelihood of using TNs, but who had used TNs with none or few clients in the past $(n=8)$; (2) clinicians with high intentions and high likelihood of using TNs who reported using TNs with all or most of the their clients in the past $(n=5)$; and (3) clinicians who reported low intentions but medium to high likelihood of using TNs who had variable levels of past TN use $(n=4)$. Of the 26 participants who completed the survey and were invited to partake in the qualitative interviews, 17 (65\%) participants completed interviews by phone or in person. Those who declined either did not respond to attempts to contact them or reported insufficient time to complete an interview. All procedures were approved by the City of Philadelphia and University of Pennsylvania Institutional Review Boards.
Semi-structured interviews focused on clinician perceptions of TNs, as well as factors that interfere with or assist their use. Several questions prompted clinicians to consider their most recent session with a client and the determinants to TN implementation in a single session [61]. These questions elicited concrete descriptions of clinicians' judgment and decision-making in order to analyze the interviews using behavioral insights (see Additional file 1 for the interview guide).

Each participant completed one interview lasting between 30 and $60 \mathrm{~min}$. The interviews were audiorecorded and conducted individually in person or by phone. BSL and HEF, both doctoral students familiar with TF-CBT and PACTS, conducted the interviews. Undergraduate research assistants transcribed the interviews. Participants received a $\$ 50$ gift card.

\section{Analytic approach}

We used an integrative approach informed by thematic analysis and a flexible adaptation of existing frameworks from the behavioral insights literature to interpret and code the qualitative data. As no single approach was sufficient to guide the hypothesis generation process, our study team integrated several guides and frameworks from the behavioral insights literature. Our analytic approach had three major phases, elaborated below.

First, in order to distill qualitative interview transcripts, thematic analysis was applied to organize the qualitative data into a manageable and interpretable amount of text [62]. Second, in order to systematize the hypothesis generation process, we selectively borrowed elements from the NUDGE framework, which has been used to design behavioral insights-derived implementation strategies based on hypothesized determinants [57]. To structure this phase, we relied heavily on the Behavioral Economics Guide to code hypothesized behavioral insights determinants of TN implementation [58]. Third, we used EAST to design behavioral insights-informed implementation strategies [59].

NUDGE is a behavioral insights approach that rigorously identifies what drives EBI implementation [57]. NUDGE lays out a multi-step process from "Narrowing" the focus to a specific behavioral target through "Understanding" the context of the behavior, "Discovering" the underlying behavioral insights, "Generating" implementation strategies, and "Evaluating" them through trials. In previous work, the NUDGE approach was used to analyze qualitative data to discover what drives EBI implementation in publicly-funded mental health agencies [57]. We adapted the "Discover" step of NUDGE into a coding process in which we applied codes for various behavioral insights largely drawn from the Behavioral Economics Guide 2018 [58]. Note that this guide is not exhaustive, and that given their training, coders were 
also familiar with other behavioral insights guides that they drew upon in this step [63]. To structure the "Generate" step of NUDGE, we used the EAST framework to propose behavioral insights-derived implementation strategies [59]. EAST was developed by the UK Behavioral Insights Team, a group of scientists and policymakers who apply findings from social psychology, cognitive science, and behavioral economics to a host of policy domains. EAST was developed as a practical and comprehensive tool for researchers and practitioners to arrange evidence in a digestible format. EAST primarily organizes behavioral insights strategies according to the principles that underlie their effectiveness. These strategies work because they make the optimal choice easier, more attractive, more social, and/or timelier than other choices. EAST offers a structured way to comprehensively consider all the mechanisms by which to address hypothesized implementation determinants.

It is important to note that, in the current study, we did not generate an exhaustive list of all potential implementation strategies. Rather, we designed several possible strategies to illustrate the promise of this structured brainstorming process.

\section{Behavioral insights coding process}

Figure 1 displays the multi-step process we used to analyze the qualitative interviews. The first phase of the coding process, described above, in which interviews were coded using thematic analysis, was conducted in Steps 1-3. The second phase of analysis, where we selectively borrowed elements from the NUDGE framework to iteratively map TN determinants onto behavioral insights using the Behavioral Economics Guide 2018 [58], was conducted in Steps 4-5. Table 1 provides definitions of the behavioral insights that we mapped onto the TN determinants. The third phase of analysis, in which we used EAST to design implementation strategies, was conducted in Step 6 [59]. See Additional file 2 for a full description of the coding process.

\section{Results \\ Demographic characteristics}

Qualitative interview participants were women $(n=17$, $100 \%)$, master's level ( $n=17,100 \%)$, predominantly licensed clinicians $(n=11,65 \%)$ with a mean age of 32.24 years $(S D=$ 9.74). The racial makeup of the sample was predominantly White ( $n=15,88 \%$ ), with other participants identifying as Black or African-American $(n=1,6 \%)$ and other $(n=1,6 \%)$. The majority identified as non-Latinx $(n=13,76 \%)$. See Table 2 for sample demographic characteristics.

Forty-one percent of participants reported using TNs with most or all of their TF-CBT clients in the past 6 months. Seventy-six percent of clinicians said it was "very likely" they would use TNs with their TF-CBT clients in the next 6 months.

\section{Major findings}

Three broad themes emerged from our analyses of clinicians' responses (see Additional file 2 for coding results and Table 3 for TN determinants, behavioral insights, and strategies).

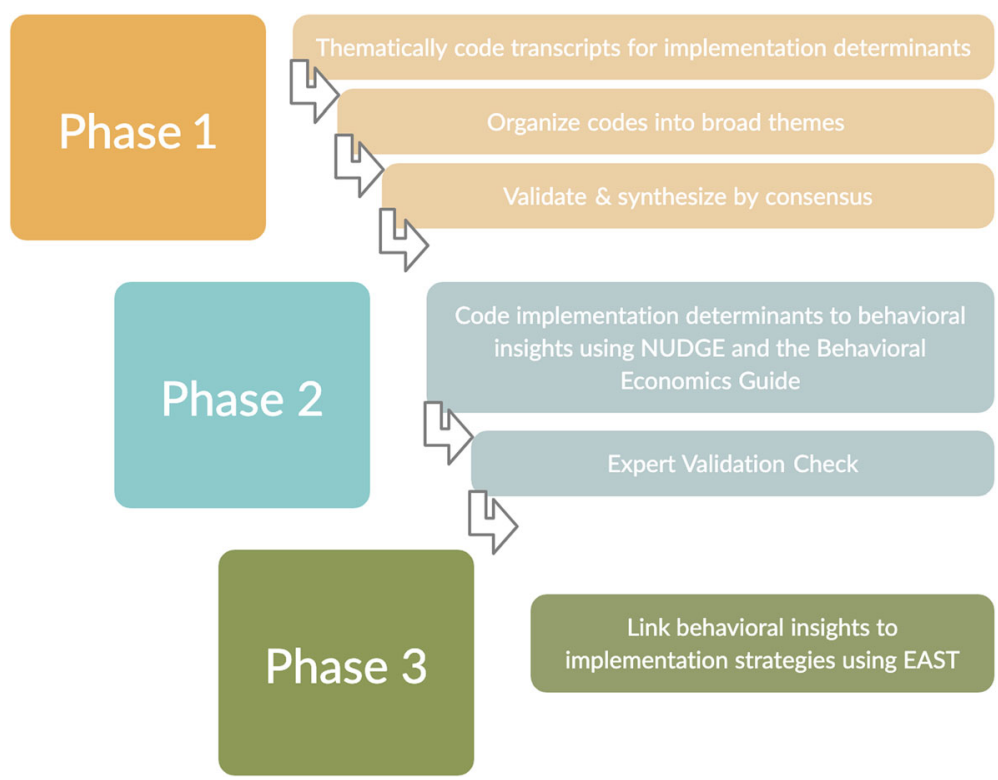

Fig. 1 Steps of the qualitative analysis process. Note. See Table 3 for details on the final themes, TN determinants, behavioral insights, and implementation strategies developed out of this process 
Table 1 Behavioral insights identified through coding process

\begin{tabular}{lll}
\hline $\begin{array}{l}\text { Behavioral } \\
\text { insights }\end{array}$ & Definition & $\begin{array}{l}\text { How behavioral insight can determine TN } \\
\text { implementation }\end{array}$ \\
\hline $\begin{array}{lll}\text { Base Rate } \\
\text { Fallacy/Mental } \\
\text { Models }\end{array}$ & $\begin{array}{l}\text { Base rate fallacy refers to when individuals ignore probabilities when } \\
\text { making decisions and instead use the similarities between events to } \\
\text { make predictions. Mental models are internal representations of the } \\
\text { world. }\end{array}$ & $\begin{array}{l}\text { Clinicians who experience the base rate fallacy may } \\
\text { believe that aggregated data from efficacy trials, which } \\
\text { are used to develop clinical practice guidelines, do not } \\
\text { apply to their individual clients because of the } \\
\text { perceived dissimilarity between their clients and trial } \\
\text { participants. TF-CBT clinicians may have mental models } \\
\text { of a "straight forward" or "typical" TF-CBT case, whereas } \\
\text { other clients may align less with their image of the } \\
\text { model of a typical TF-CBT case. }\end{array}$
\end{tabular}

\begin{abstract}
Choice
Overload/

Decision Fatigue

Choice overload occurs when decision-makers are faced with too many choices-the more choices, the more likely decision-makers will employ heuristics in lieu of reason. This relates to decision fatigue, or when people become fatigued the more decisions they make, which leads to poorer decisions.
\end{abstract}

Default Bias

Default bias is the tendency for decision-makers to prefer the current state of affairs and an aversion to change.

Fear Avoidance/ Ostrich Effect

ear avoidance is the tendency to avoid thoughts or actions that cause people fear. The ostrich effect is related to fear avoidance; it describes people's tendency to ignore or fail to seek, often negative, information.

Functional Fixedness

Hopelessness/ Helplessness

\begin{abstract}
Lack of Reinforcement

The lack of reinforcement is the absence of a reward that can strengthen a response or action.
Risk/Loss
Aversion
Social Norms gains. This leads people to avoid risks when losses are involved.
Social norms represent a psychological phenomenon in which people do something primarily because other people like them are doing it.

Loss aversion refers to the idea that losses are more painful than similar
\end{abstract}

Hopelessness and helplessness are the feelings that things will not get better and that there are no ways to improve the situation.

Functional fixedness is the tendency to conceptualize an object (broadly construed) only in terms of its most common use.
Clinicians may feel that they don't know how to choose among the many different intervention options (i.e., modality of the narrative, how to structure the narrative, etc.) they have at their disposal for a given client. They may feel psychologically taxed by the multiple decisions.

Clinicians may prefer the current practices they implement in their clinical work. This occurs because the current treatments they are implementing are taken as a reference point, and any change from that baseline is perceived as less preferable.

Clinicians may avoid implementing the trauma narrative because it is difficult for them-they may not be as skilled in the trauma narrative as the practices they have been trained in, and therefore do not want to engage in something that makes them feel less competent or nervous. They may also fear the difficulty in hearing details they may learn during the trauma narrative.

Clinicians may believe that the trauma narrative can only be done in the way that it has been taught to them. For example, if a clinician is only taught to implement the trauma narrative verbally, they may struggle to consider other methods/modalities by which to implement it.

Clinicians may feel "stuck" when attempting to implement the trauma narrative because several other barriers or challenges have intervened their ability to implement it. Clinicians may feel that despite their attempts to implement the trauma narrative, due to factors outside of their control (e.g., the client's psychosocial stressors, their inability to attend sessions) they are being insufficiently rewarded for their work, and therefore may be less inclined to attempt it with some clients.

Clinicians may feel they are not being rewarded for the uncompensated work they have to do to prepare for the trauma narrative session.

Clinicians may perceive the risk of harm in conducting the trauma narrative as more salient than the benefits it may offer.

Clinicians may feel that if others at their agency are/are not using the trauma narrative, then they will be less/ more likely to use it.

Note. Behavioral insights are largely selected from the Behavioral Economics Guide 2018 [58] with several additions included by study coders with expertise in behavioral insights

\section{Decision complexity}

Decision complexity refers to the dimensions of a decision problem. The more dimensions of the problem, the more complex it is [64]. Behavioral insights suggest that more complex decisions lead clinicians to take longer to decide, to make more errors when they do, and to feel less confident in their decisions [65].

TN determinants Clinicians who were overwhelmed by the complexity (e.g., client psychosocial and symptom 
Table 2 Demographic characteristics

\begin{tabular}{|c|c|}
\hline Characteristic & $\mathrm{N}(\%)$ or Mean (SD, range) \\
\hline Age & $32.24(9.74,21-62)$ \\
\hline Gender (Woman) & 17 (100\%) \\
\hline \multicolumn{2}{|l|}{ Hispanic/Latinx } \\
\hline Yes & $4(23.53 \%)$ \\
\hline \multicolumn{2}{|l|}{ Race } \\
\hline American Indian/Alaskan Native & 0 \\
\hline Asian & 0 \\
\hline Black or African American & $1(5.88 \%)$ \\
\hline Native Hawaiian or Pacific Islander & 0 \\
\hline White & $15(88.24 \%)$ \\
\hline Other & $1(5.88 \%)$ \\
\hline \multicolumn{2}{|l|}{ Licensed } \\
\hline Yes & $11(64.71 \%)$ \\
\hline \multicolumn{2}{|l|}{ Which License? } \\
\hline Professional Counselor & $2(11.76 \%)$ \\
\hline Clinical Social Worker & $5(29.41 \%)$ \\
\hline Licensed Social Worker & $3(17.65 \%)$ \\
\hline Marriage and Family Therapy & $1(5.88 \%)$ \\
\hline Not Licensed & $6(35.29 \%)$ \\
\hline \multicolumn{2}{|l|}{ Highest Degree? } \\
\hline MA & $4(23.53 \%)$ \\
\hline MS & $4(23.53 \%)$ \\
\hline MEd & $1(5.88 \%)$ \\
\hline MSW & $6(35.29 \%)$ \\
\hline DSW & $1(5.88 \%)$ \\
\hline MSS & $1(5.88 \%)$ \\
\hline \multicolumn{2}{|l|}{ Profession } \\
\hline Social Worker & $8(47.06 \%)$ \\
\hline Professional Counselor & 9 (52.94\%) \\
\hline Years Practicing & $6.00(7.84,0.83-31.17)$ \\
\hline \multicolumn{2}{|l|}{ Completion of PACTS Training } \\
\hline 2011 & $1(5.88 \%)$ \\
\hline 2015 & $3(17.65 \%)$ \\
\hline 2016 & $2(11.76 \%)$ \\
\hline 2017 & $4(23.53 \%)$ \\
\hline 2018 & $6(35.29 \%)$ \\
\hline No Response & $1(5.88 \%)$ \\
\hline
\end{tabular}

Note. Many clinicians in public mental health settings work under the license of their supervisor (often a licensed social worker or licensed professional counselor)

complexity, client developmental level, and the variety of therapeutic techniques available) cited it as a major barrier to TN use. They described a high level of uncertainty once several features of their clients did not map onto their schema of a typical TF-CBT client.
Conversely, other clinicians were able to reduce the complexity of decisions through processes like staging (i.e., breaking the decision up into its essential parts) or using decision aids [66]. Clinicians experienced in other EBIs described their skills as an asset, embracing the flexibility of the model.

Behavioral insights The TN determinants revealed several behavioral insights: choice overload/decision fatigue, base rate fallacy/mental models, and functional fixedness. Choice overload is a cognitive process in which people have difficulty making a decision when faced with many options. This phenomenon is related to decision fatigue, which describes how the more decisions clinicians make, the poorer their clinical judgement [67]. When clinicians encounter clients with severe psychopathology, psychosocial stressors, and other challenges, they feel overloaded or fatigued. Other clinicians reported strategies such as accepting that TNs would not solve all of their clients' problems, reframing their goals, or reducing their choices.

Clinicians who described that certain clients were better suited to creating TNs were potentially committing the base rate fallacy and revealed their specific mental models. The base rate fallacy arises when clinicians believe that aggregated data do not apply to individual clients. Mental models are people's internal representations of a problem. Clinicians revealed that their vision of a "straight-forward" TF-CBT case is different from the cases they see. Functional fixedness captures clinicians' perception that TNs can only be expressed in written form - the particular way they were trained to implement TNs. This prevents clinicians from integrating other clinical skills that would facilitate recovery. Clinicians who incorporated other techniques understood the purpose of the TN as a therapeutic tool beyond understanding how it is regularly implemented.

Implementation strategies We used EAST to develop an implementation strategy that would disrupt clinicians' mental models and functional fixedness. Showing clinicians that peers working in similar contexts can use techniques from other EBIs (e.g., evidence-based play therapy) may prompt clinicians to have more flexible mental models while at the same time providing a leading example for how other EBIs can be incorporated into the implementation of TNs $[68,69]$. This would enable clinicians who are more flexible to influence those who are less flexible. This strategy would involve clinicians who incorporate other EBIs into TNs distributing stories or descriptive guides.

For clinicians who believe that certain client characteristics make TNs easier/harder, revealing mental models and choice overload, we generated a strategy in which 


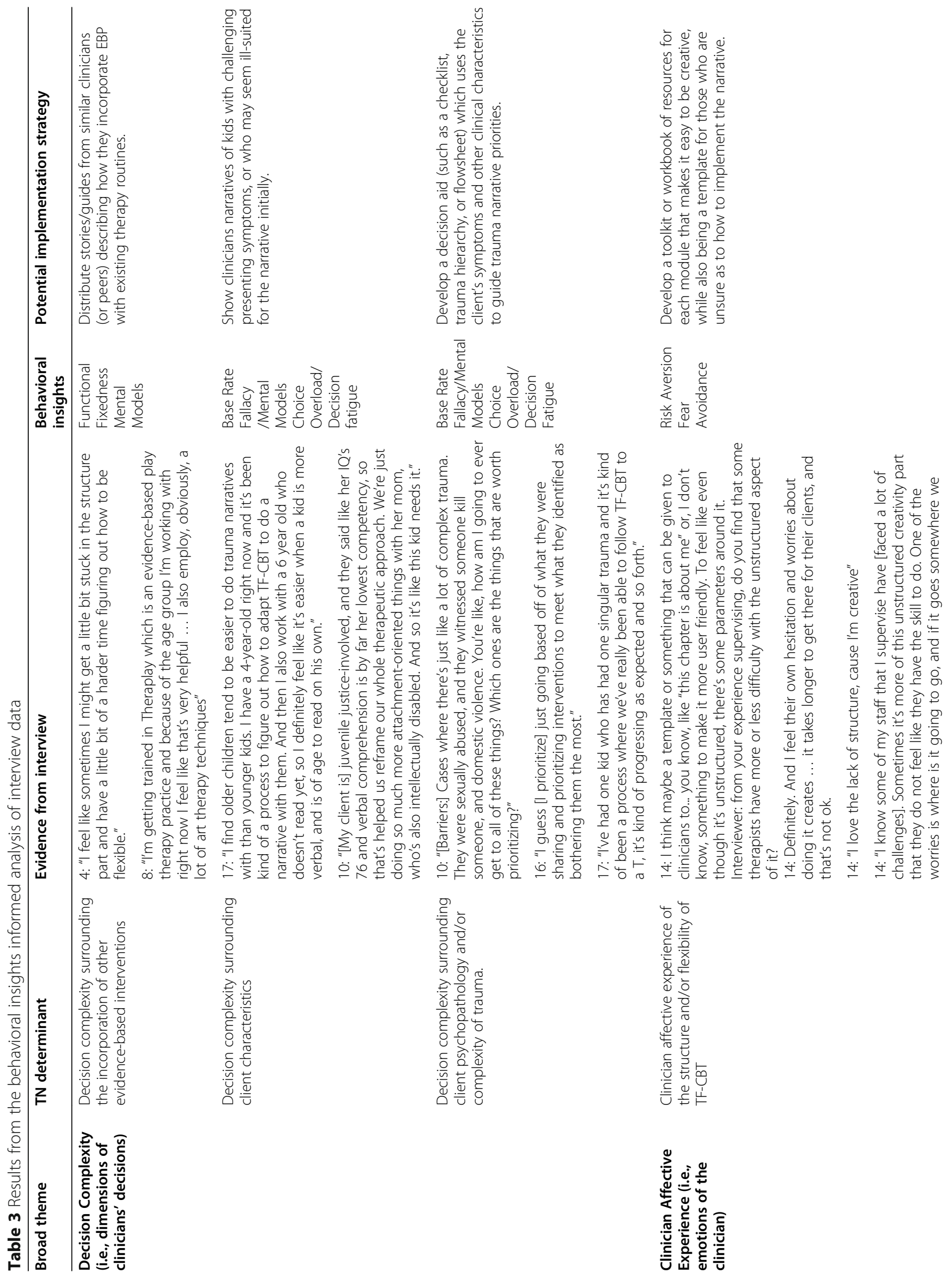




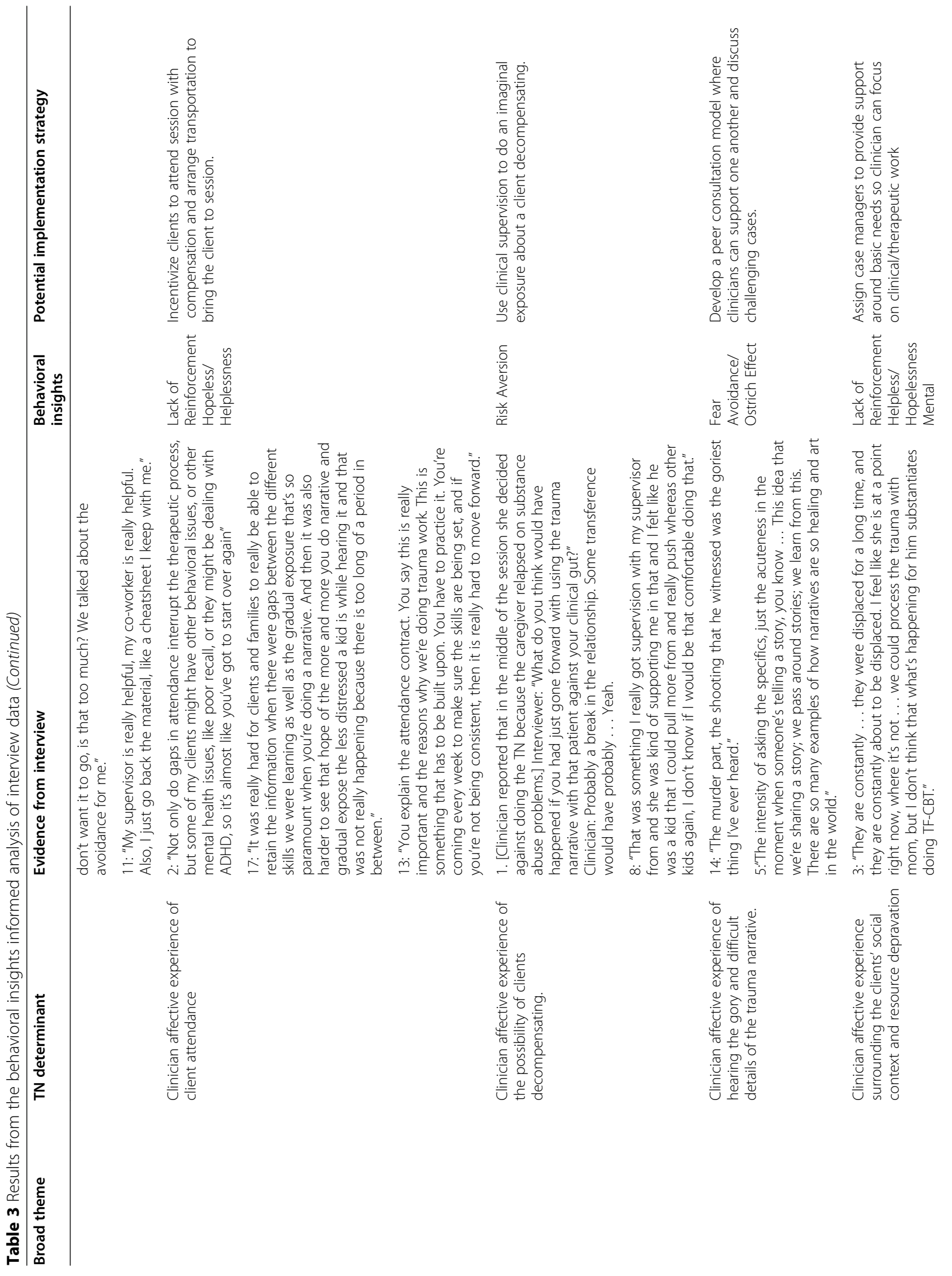




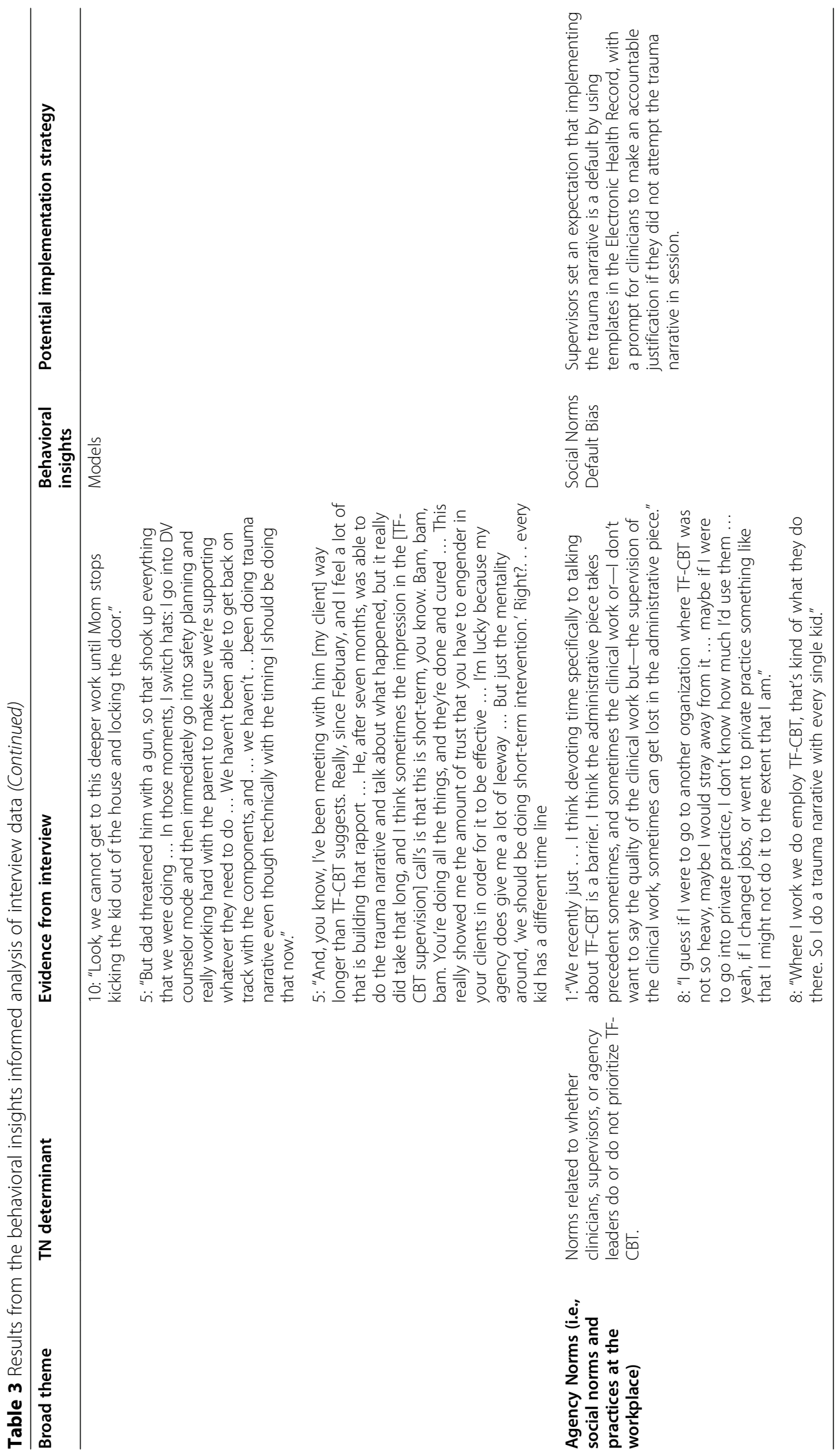


supervisors show clinicians narratives of clients with challenging presenting symptoms, or who may seem illsuited for TNs initially. This would provide a blueprint for clinicians with challenging clients. For clinicians who are concerned about their clients having their basic needs met, feeling helpless/hopeless, we designed a strategy to ease their burden. For clinicians with choice overload/decision fatigue relating to their clients' severe psychopathology, we proposed a decision aid (such as a checklist, trauma hierarchy, or flowsheet) which uses the client's clinical characteristics to guide TN priorities. Decision aids are behavioral insights-informed strategies for choice overload/decision fatigue [66].

\section{Affective experience}

Invariably, implementing psychological EBIs can provoke intense emotions. Trauma therapy is well known to cause clinicians distress. These emotions can, in turn, influence the quality of clinical decisions [70-72]. Evidence also suggests that clinicians working in high poverty contexts tend to experience additional stress given the enormous needs of their clients and the feeling of powerlessness this can engender [73].

TN determinants Some clinicians described feeling overwhelmed by their clients' economic hardships or by their clinical severity. Other clinicians described feeling distressed listening to graphic TNs or feeling afraid to push clients too far. Some indicated that the model is insufficiently concrete, leading them to feel anxious and uncertain. Many described not feeling rewarded for their uncompensated session planning and losing hope in clients' improvement due to long treatment gaps or family disengagement. Contrary to clinicians who reported feeling overwhelmed by TNs (either due to their flexibility or their content), other clinicians reporting seeking guidance and support from their supervisors and reframing their perspective about TNs. Clinicians who might feel disappointed by inconsistent attendance instead created rules to ensure that clients would consistently attend.

Behavioral insights These determinants revealed several behavioral insights: risk/loss aversion, fear avoidance/ostrich effect, lack of reinforcement, helplessness/hopelessness, base rate fallacy/mental models, and functional fixedness. Risk/loss aversion is the tendency to prefer avoiding losses to acquiring similar gains. Clinicians may perceive the risk of conducting TNs as more salient than the benefits they offer. Fear avoidance is the tendency to avoid thoughts or actions that cause people fear. The ostrich effect is a related phenomenon; it describes people's tendency to ignore obvious, often negative, information because it is inconvenient or anxiety-inducing. Clinicians may avoid implementing TNs because they are difficult-clinicians may not be as skilled in TN delivery as they are in other practices. Clinicians may fear doing something that makes them feel incompetent. Some described dreading TN details because they are graphic and potentially produce vicarious traumatization.

Positive reinforcement describes the increased frequency of behaviors when they result in rewards [74]. Some clinicians described not feeling rewarded for their work, specifically for uncompensated TN preparation (e.g., session planning), as well as for their sustained attempts to help clients whose treatment was often derailed by more acute needs (e.g., psychosocial stressors). Despite clinicians' attempts to implement TNs, due to factors outside of their control (such as clients' crises that lead to missing sessions), they described feeling insufficiently rewarded-i.e., clients were not getting better. This lack of reinforcement may have led them to feel less inclined to attempt to implement TNs. Persistent lack of reinforcement led clinicians to experience helplessness and hopelessness about their clients' progress and disappointment that TNs were not a panacea. Some clinicians avoided experiencing the lack of reinforcement, helplessness, and hopelessness by managing their expectations and reframing their goals.

Clinicians who described being able to manage their expectations and goals for clients viewed TNs as easier to implement, and displayed less risk/loss aversion, fear avoidance, and helplessness/hopelessness. They understood that they could not solve everything in their clients' lives, which may have allowed them to reframe their expectations and mitigate the potential lack of reinforcement. Some clinicians reported seeking support and encouragement from their supervisors, reaffirming the rationale of TNs to themselves, and planning ahead to ensure that clients did not consistently lose momentum. Clinicians' strategies to seek positive reinforcement from their supervisors/agencies enabled them to feel rewarded for their efforts irrespective of the forces outside of their control.

Implementation strategies For clinicians who reported anxiety about the flexibility of the narrative, we generated an implementation strategy that would prevent this anxiety and provide concrete assistance to narrow the possibilities. We suggested the development of a toolkit or workbook of resources for TNs, serving as both a template and a toolkit of creative ideas. Some TF-CBT clinicians cited already using templates as helpful in alleviating their anxiety. Given that this anxiety appears to stem from an intolerance of uncertainty, providing concrete tools for clinicians can assuage their worries [75].

For clinicians who reported losing momentum due to clients' inconsistent attendance, we developed a strategy 
that would reduce the frustration and worries of clinicians by incentivizing clients to attend session with financial compensation and arranged transportation. This would indirectly address the affective experience of clinicians by making it less likely that clients miss sessions. For clinicians who experience significant emotional distress about TNs (i.e., worrying that clients will decompensate or that the details will be difficult for them to hear), we generated implementation strategies to directly address clinicians' anxieties through supportive techniques. One strategy involves using clinical supervision more therapeutically, acknowledging that clinicians also experience secondary traumatic stress. One technique that can be employed in group supervision is to do an imaginal exposure to feared outcomes (e.g., a client decompensating), effectively treating clinicians' anxieties [76, 77]. We also generated a peer consultation model strategy where clinicians can support one another and discuss challenging cases. These practices would be incorporated into the supervision model (creating a default) which would reduce the effort of clinicians to seek support independently. The social element of the supervision and consultation models would make it more likely that clinicians feel supported and not alone. Assigning a case manager to provide support around clients' basic needs would enable clinicians to focus on their therapeutic work and eliminate their worries that they should be prioritizing non-therapeutic casework. Equipped with the knowledge that their clients would be cared for, this strategy would help clinicians feel less hopeless about their clients' prospects.

\section{Agency norms}

The final broad theme was agency norms-the social norms of clinicians' agency leaders, supervisors, and peers. Evidence suggests that social norms strongly influence behavior [78].

TN determinants Clinicians reported that if it was standard practice to use TNs in their agencies, clinicians would employ TNs. When agency leaders, supervisors, and colleagues did not prioritize TNs, clinicians reported that they were less likely to use TNs.

Behavioral insights Agency norms reveal the behavioral insight that clinicians are influenced by the default bias and social norms. Clinicians prefer the current state of affairs, or the default practices they typically use in their clinical work. This default is taken as a reference point, and any change from that baseline is perceived as less preferable and sometimes aversive. Social norms arise when people do something primarily because others like them do. Clinicians are influenced by others at their agency who do or do not use TNs.
Implementation strategies To address social norms and default bias, we generated an implementation strategy that makes use of the electronic health records clinicians typically use to record progress notes. Agencies and supervisors would create templates in the electronic health record that would require clinicians to describe their attempts to implement TNs. Clinicians would be prompted to write a justification if they did not attempt a TN in session with the knowledge that their supervisors would see the note. Establishing a default ensures that the standard practice is to use TNs, and, further, it creates a social norm that everyone at the agency implements TNs. Strategies prompting clinicians to provide justification embedded in electronic health records have been effective at increasing the use of other EBIs in medical settings [79].

\section{Discussion}

Our study combines an in-depth qualitative analysis and a systematic application of theoretical principles from behavioral insights to understand implementation of an effective EBI for youth with PTSD [57]. We interviewed clinicians to identify implementation determinants of a core component of TF-CBT, the TN. We generated novel implementation strategies to target the hypothesized behavioral insights determining implementation behavior. The study identified three major themes relating to why clinicians do or do not use TNs: (1) decision complexity, (2) affective experience, and (3) agency norms. First, clinicians working in public mental health settings feel they are faced with particularly complex clients and contexts and have trouble translating clinical guidelines to practice. We generated implementation strategies that reduce decision complexity through decision aids and offloading responsibilities from clinicians. Second, the affective experience of clinicians implementing TNs in resource-scarce environments with severe clients leads them to feel overwhelmed and anxious. Clinicians' experiences can be targeted through anxiety prevention strategies and therapeutic and emotionally supportive practices at the organizational level. Third, agency norms reflect clinician perceptions of what is considered standard practice in their agencies and determines TN use. The behavioral insights-informed strategy involves changing agency practices to facilitate TN implementation.

These results broaden our understanding of EBI implementation by analyzing clinicians' lived experiences with theories on judgment and decision-making to design targeted and novel implementation strategies. Our study suggests that behavioral insights can provide a coherent theoretical guide across the implementation research continuum (from identifying determinants to practical guidance for implementation strategy design 
and selection) [80, 81]. This approach allowed us to go beyond the face-value understanding of clinicians' firstperson accounts and to develop hypotheses about the behavioral insights that may explain both clinicians' behavior and clinicians' understanding of their own implementation behavior.

One advantage of extending qualitative data beyond their immediate and literal meaning, particularly through the use of behavioral insights theories, can be demonstrated by example. Some clinicians in our study reported that their clients had more complex and frequent traumas than "most" clients-presumably than the typical child seeking trauma therapy. This barrier, if read literally, might be coded as "clinician knowledge and beliefs" under other widely used implementation frameworks [82]. Analyzing our data using behavioral insights offers us an additional lens through which to understand implementation behavior. We determined that this commonly voiced refrain may instead reveal clinicians' choice overload [83]. When faced with an overwhelming amount of information, individual decision-makers tend to give up on their intended behaviors and offer posthoc rationalizations for why they did not engage in them. Clinicians in public mental health settings likely encounter more severe and more complex clients than TF-CBT RCT participants-indeed, we have data to support this belief in Philadelphia [84]. However, little data support the notion that more severe clients would not benefit from the $\mathrm{TN}$, though their symptoms may not fully remit [85]. The hypothesis that choice overload is the underlying psychological driver of clinicians' behavior does not map onto an attitudinal implementation strategy; rather it maps onto designing a decision aid to distill a complex decision into a simpler, more digestible format. Decision aids are known to reduce complexity and simplify clinical decisions to optimize and improve clinical judgment [86]. Through using this behavioral insights-informed approach, we generated hypotheses about what drives implementation behavior based on what is latent in the qualitative data, but not literally stated by clinicians. This interpretative leap has its pitfalls-the hypothesized determinant may not apply-but we can justify our understanding through the extensive empirical literature that validate behavioral insights. In future research, the behavioral insights-informed implementation strategies can be evaluated for their effectiveness and our hypotheses about the behavioral insights serving as the mediating pathways can be tested.

Our study fits well within the literature on TF-CBT implementation. TF-CBT has been disseminated and implemented through various methods including remote web-based learning, live training, ongoing phone consultation, learning collaborative models, and some combination therein [87-90]. These efforts have been undertaken in a variety of public mental health settings in the USA and in low- and middle-income countries around the world [91-93]. Many of these studies' results are consistent with our findings on clinicians' self-reports of the challenges to TF-CBT implementation. For example, clinicians across settings believe that their contexts are quite different from the contexts of RCT trial participants. Our work also validates research from TF-CBT national trainers that TN adherence is low [17]. Thus, our work's focus on clinicians' perspectives of TNs, considered an active component of TF-CBT by treatment developers and national trainers, is an essential contribution to TF-CBT implementation research.

\section{Limitations}

There were several limitations to our study. First, our analysis was based on clinician self-report. There is an inherent tension between attempting to discover the often unconscious psychological drivers of TN implementation and relying on clinicians' self-report [94]. For example, data from the national trainers suggests that general psychotherapy competence is a core challenge for TF-CBT implementation [17]. Yet, none of the study participants described feeling incompetent, potentially confirming the behavioral insight that people prefer attributions that are self-enhancing over those that are self-deprecating [95]. Though qualitative data were analyzed beyond their immediate meaning, complementary quantitative measures of clinicians' behavior (e.g., effectiveness and skill) were not collected that would have provided more data to understand implementation determinants.

Second, our approach primarily addresses individual clinicians' decisions. Though we generated implementation strategies that target organizations (e.g., developing peer consultation models, transforming the electronic health record, hiring case managers, etc.), behavioral insights are less well suited to address organizational challenges or structural barriers (e.g., scarcity of resources) of which there are many in publicly funded mental health systems [7, 96]. Studying mental healthcare delivery calls for attention not just to individual clinician decisions, but to a structural understanding that takes all levels of analysis into account $[1,97]$. Public mental health agencies in Philadelphia have benefited from a fertile policymaking ecology, which has incentivized EBI implementation. How policy decisions interact with outer context and individual clinician decision determinants was not explicitly explored, though clinicians often described these complex interactions. Ultimately, all solutions to serious public mental health concerns involve transforming individual behavior as the final link in the chain, making the analysis of judgment and decision-making critical. Future work should 
uncover both the potential and the limits inherent to examining individual clinician decisions as the unit of analysis to understand EBI implementation.

\section{Conclusions}

In-depth qualitative interviews revealed that clinicians implementing TNs-an active component of an effective EBI, TF-CBT-in public mental health agencies are faced with challenges relating to decision complexity, their affective experiences, and agency norms. We generated behavioral insights informed by hypotheses about what determines clinicians' implementation behavior and designed corresponding implementation strategies using an established behavioral insights framework (EAST). Future research will test these implementation strategies to understand if and how they work. This work will also integrate the behavioral insights discovered from this approach with insights from implementation science frameworks that account for structural and organizational contexts. The goal of our work is to synthesize interdisciplinary knowledge to determine the factors that impede and facilitate EBI implementation, and to test methods to improve implementation. Understanding clinical judgment and decision-making will enhance our capacity to design effective approaches to improve healthcare.

\section{Supplementary Information}

The online version contains supplementary material available at https://doi. org/10.1186/s43058-020-00105-6.

Additional file 1. Qualitative Interview Guide.

Additional file 2. Behavioral Insights Coding Process and Results.

\section{Abbreviations}

DBHIDS: Department of Behavioral Health and Intellectual Disability Services; EAST: Easy Attractive Social and Timely; EBI: Evidence-based Intervention; NUDGE: Narrow, Understand, Discover, Generate, and Evaluate framework; PACTS: Philadelphia Alliance for Child Trauma Services; TF-CBT: TraumaFocused Cognitive Behavioral Therapy; TN: Trauma Narrative

\section{Acknowledgements}

We are grateful for the support and partnership that the Department of Behavioral Health and Intellectual Disability Services (DBHIDS) provided for this project and for the Evidence-Based Practice and Innovation Center (EPIC). We are also very grateful to the individuals who have been a part of the Philadelphia Alliance for Child Trauma Services (PACTS), including DBHIDS and Community Behavioral Health leadership, the clinicians, administrators, and families who have been involved. We are grateful to Jessica Fishman, PhD, for her contributions to this study.

\section{Authors' contributions}

BSL conceived of and designed the research study, acquired and analyzed the data, interpreted the data, drafted the manuscript, and substantially revised it. SHS analyzed the data, interpreted the data, and substantially revised the manuscript. CET analyzed the data, interpreted the data, and substantially revised the manuscript. HEF helped design the research study, acquired and analyzed the data, and substantially revised the manuscript. AMB helped design the research study, analyzed the data, interpreted the data, and substantially revised the manuscript. BNR acquired the data and substantially revised the manuscript. SFM substantially revised the manuscript. CC substantially revised the manuscript. SS substantially revised the manuscript. RSB helped conceive of and design the research study, interpreted the data, and substantially revised the manuscript. The authors approved the submitted version, have agreed to be accountable for the contributions, and attest to the accuracy and integrity of the work, even aspects for which the authors were not personally involved.

\section{Funding}

Funding for this study was provided by grants from the Substance Abuse and Mental Health Services Administration (SM063192, Beidas, Subaward PI) and the National Institute of Mental Health (F31MH112211, Frank, Pl; T32 MH109433 Rudd), Beidas, Mandell, MPI; P50 MH 113840, Beidas, Buttenheim, Mandell, MPI). Additional funding was awarded to Briana S. Last from the University of Pennsylvania, Psychology Department. Briana S. Last also receives funding support from the National Science Foundation Graduate Research Fellowship Program (DGE-1321851).

\section{Availability of data and materials}

The dataset generated and analyzed during the current study is not publicly available due to the highly sensitive nature of interview transcript data. Publication of entire transcripts risk identifying research participants.

\section{Ethics approval and consent to participate}

All procedures were approved by the City of Philadelphia (2012-47) and the University of Pennsylvania (817282) Institutional Review Boards. All participants provided written or verbal consent to participate in our research study.

\section{Consent for publication}

Not applicable.

\section{Competing interests}

The authors declare no financial or non-financial competing interests.

\section{Author details}

${ }^{1}$ Department of Psychology, University of Pennsylvania, Philadelphia, PA, USA. ${ }^{2}$ College of Liberal and Professional Studies, University of Pennsylvania, Philadelphia, PA, USA. ${ }^{3}$ Department of Psychology, Temple University, Philadelphia, PA, USA. ${ }^{4}$ Department of Psychiatry and Human Behavior, Warren Alpert Medical School of Brown University, Providence, RI, USA. ${ }^{5}$ Department of Family and Community Health, School of Nursing, University of Pennsylvania, Philadelphia, PA, USA. ${ }^{6}$ Center for Health Incentives and Behavioral Economics (CHIBE), University of Pennsylvania, Philadelphia, PA, USA. ${ }^{7}$ Penn Implementation Science Center at the Leonard Davis Institute of Health Economics (PISCE@LDI), University of Pennsylvania, Philadelphia, PA, USA. ${ }^{8}$ Department of Psychiatry, University of Pennsylvania Perelman School of Medicine, Philadelphia, PA, USA. ${ }^{9}$ Department of Psychiatry, University of Illinois at Chicago, Chicago, IL, USA. ${ }^{10}$ Community Behavioral Health, Philadelphia, PA, USA. "11Department of Behavioral Health and Intellectual Disability Services, Philadelphia, PA, USA. ${ }^{12}$ Department of Medical Ethics and Health Policy, University of Pennsylvania Perelman School of Medicine, Philadelphia, PA, USA. ${ }^{13}$ Department of Medicine, University of Pennsylvania Perelman School of Medicine, Philadelphia, PA, USA.

Received: 17 August 2020 Accepted: 17 December 2020

Published online: 11 January 2021

\section{References}

1. Raghavan R, Bright CL, Shadoin AL. Toward a policy ecology of implementation of evidence-based practices in public mental health settings. Implement Sci. 2008;3(1):26.

2. APA Presidential Task Force on Evidence-Based Practice. Evidence-based practice in psychology. Am Psychol. 2006;61(4):271-8.

3. Dickman SL, Himmelstein DU, McCormick D, Woolhandler S. Health and financial consequences of 24 states' decision to opt out of Medicaid expansion. Int J Health Serv. 2015;45(1):133-42.

4. Dickman SL, Himmelstein DU, Woolhandler S. Inequality and the health-care system in the USA. Lancet. 2017;389(10077):1431-41.

5. Aizer $\mathrm{A}$, Currie J. The intergenerational transmission of inequality: maternal disadvantage and health at birth. Science. 2014;344(6186):856-61. 
6. Stewart RE, Adams DR, Mandell DS, Hadley TR, Evans AC, Rubin R, et al. The perfect storm: collision of the business of mental health and the implementation of evidence-based practices. Psychiatric Serv. 2016;67(2):159-61.

7. Beidas RS, Marcus S, Aarons GA, Hoagwood KE, Schoenwald S, Evans AC, et al. Individual and organizational factors related to community clinicians' use of therapy techniques in a large public mental health system. JAMA Pediatr. 2015;169(4):374-82

8. Aarons GA, Wells RS, Zagursky K, Fettes DL, Palinkas LA. Implementing evidence-based practice in community mental health agencies: a multiple stakeholder analysis. Am J Public Health. 2009;99(11):2087-95.

9. Corrigan PW, Watson AC. Factors that explain how policy makers distribute resources to mental health services. Psychiatric Serv. 2003;54(4):501-7.

10. Borkovec TD, Costonguay LG. What is the scientific meaning of empirically supported therapy? I Consult Clin Psychol. 1998;66(1):136.

11. Hofmann SG, Hayes SC. The future of intervention science: process-based therapy. Clin Psychol Sci. 2019;7(1):37-50.

12. Weisz J, Bearman SK, Santucci LC, Jensen-Doss A. Initial test of a principleguided approach to transdiagnostic psychotherapy with children and adolescents. J Clin Child Adolesc Psychol. 2017;46(1):44-58.

13. Wolk CB, Becker-Haimes EM, Fishman J, Affrunti NW, Mandell DS, Creed TA. Variability in clinician intentions to implement specific cognitive-behavioral therapy components. BMC Psychiatry. 2019;19(1):406.

14. England MJ, Butler AS, Gonzalez ML. Psychosocial interventions for mental and substance use disorders: a framework for establishing evidence-based standards. Washington (DC): National Academies Press (US); 2015.

15. Kaye JL. Targeting therapists' experiential avoidance during exposure delivery: an experimental investigation to improve the quality of exposurebased interventions. Philadelphia: Drexel University; 201.

16. Becker-Haimes EM, Okamura KH, Wolk CB, Rubin R, Evans AC, Beidas RS. Predictors of clinician use of exposure therapy in community mental health settings. J Anxiety Disord. 2017;49:88-94.

17. Hanson RF, Gros KS, Davidson TM, Barr S, Cohen J, Deblinger E, et al. National trainers' perspectives on challenges to implementation of an empiricallysupported mental health treatment. Adm Policy Ment Health. 2014;41(4):522-34.

18. Beidas RS, Becker-Haimes EM, Adams DR, Skriner L, Stewart RE, Wolk CB, et al. Feasibility and acceptability of two incentive-based implementation strategies for mental health therapists implementing cognitive-behavioral therapy: a pilot study to inform a randomized controlled trial. Implement Sci. 2017;12(1):148.

19. Kazdin AE. Evidence-based treatment and practice: new opportunities to bridge clinical research and practice, enhance the knowledge base, and improve patient care. Am Psychol. 2008;63(3):146.

20. Southam-Gerow MA, Dorsey S. Qualitative and mixed methods research in dissemination and implementation science: introduction to the special issue. J Clin Child Adolesc Psychol. 2014;43(6):845-50.

21. Powell BJ, Beidas RS, Lewis CC, Aarons GA, McMillen JC, Proctor EK, et al. Methods to improve the selection and tailoring of implementation strategies. J Behav Health Serv Res. 2017;44(2):177-94.

22. Rousseau DM, Tijoriwala SA. What's a good reason to change? Motivated reasoning and social accounts in promoting organizational change. J App Psychol. 1999;84(4):514.

23. Hallsworth M, Kirkman E. Behavioral insights. Cambridge: MIT Press; 2020.

24. Thaler RH. Behavioral economics: past, present, and future. Am Econ Rev. 2016;106(7):1577-600.

25. Simon HA. Models of bounded rationality: empirically grounded economic reason. Vol. 3. Cambridge: MIT press; 1997.

26. Sanna $\sqcup$, Schwarz N. Metacognitive experiences and human judgment: the case of hindsight bias and its debiasing. Curr Direct Psychol Sci. 2006;15(4): 172-6.

27. Thaler RH, Sunstein CR. Nudge: improving decisions about health, wealth, and happiness. Revised and Expanded Edition. New York: Penguin Books; 2009.

28. Kahneman D, Knetsch JL, Thaler RH. Anomalies: the endowment effect, loss aversion, and status quo bias. J Econ Perspect. 1991;5(1):193-206.

29. Jachimowicz JM, Duncan S, Weber EU, Johnson EJ. When and why defaults influence decisions: a meta-analysis of default effects. Behav Public Policy. 2019;3(2):159-86.

30. Ansher C, Ariely D, Nagler A, Rudd M, Schwartz J, Shah A. Better medicine by default. Med Decis Making. 2014;34(2):147-58.

31. Loeb KL, Radnitz C, Keller K, Schwartz MB, Marcus S, Pierson RN, et al. The application of defaults to optimize parents' health-based choices for children. Appetite. 2017;113:368-75.
32. Fishbach A, Eyal T, Finkelstein SR. How positive and negative feedback motivate goal pursuit. Soc Person Psychol Compass. 2010;4(8):517-30.

33. Michie S, van Stralen MM, West R. The behaviour change wheel: a new method for characterising and designing behaviour change interventions. Implement Sci. 2011;6. https://doi.org/10.1186/1748-5908-6-1.

34. Vallerand RJ, Reid G. On the relative effects of positive and negative verbal feedback on males' and females' intrinsic motivation. Can J Behav Sci Revue canadienne des sciences du comportement. 1988;20(3):239.

35. Anderson BA. Social reward shapes attentional biases. Cogn Neurosci. 2016; 7(1-4):30-6.

36. Mbindyo P, Gilson L, Blaauw D, English M. Contextual influences on health worker motivation in district hospitals in Kenya. Implement Sci. 2009;4(1):1-10.

37. Eccles JS, Wigfield A. Motivational beliefs, values, and goals. Ann Rev Psychol. 2002;53(1):109-32.

38. Bohren MA, Hofmeyr GJ, Sakala C, Fukuzawa RK, Cuthbert A. Continuous support for women during childbirth. Cochrane Database Syst Rev. 2017; 7(7):CD003766. Published 2017 Jul 6. https://doi.org/10.1002/14651858. CD003766.pub6.

39. Potthoff S, Presseau J, Sniehotta FF, Johnston M, Elovainio M, Avery L. Planning to be routine: habit as a mediator of the planning-behaviour relationship in healthcare professionals. Implement Sci. 2017;12(1):24.

40. Potthoff S, Rasul O, Sniehotta FF, Marques M, Beyer F, Thomson R, et al. The relationship between habit and healthcare professional behaviour in clinical practice: a systematic review and meta-analysis. Health Psychol Rev. 2019; 13(1):73-90.

41. Thomson A, Vallee-Tourangeau G, Suggs LS. Strategies to increase vaccine acceptance and uptake: from behavioral insights to context-specific, culturally-appropriate, evidence-based communications and interventions. Vaccine. 2018;36(44):6457-8.

42. Buttenheim AM, Fiks AG, Burson RC 2nd, Wang E, Coffin SE, Metlay JP, et al. A behavioral economics intervention to increase pertussis vaccination among infant caregivers: a randomized feasibility trial. Vaccine. 2016;34(6): $839-45$

43. Betsch C, Böhm R, Chapman GB. Using behavioral insights to increase vaccination policy effectiveness. Policy Insights Behav Brain Sci. 2015;2(1):61-73.

44. OECD. Behavioural insights and public policy: lessons from around the world. Paris: OECD Publishing; 2017. https:/doi.org/10.1787/9789264270480-en.

45. World Health Organization. Behavioural Insights. World Health Organization. (Science Division). Available from: https://www.who.int/departments/ science-division/behavioural-insights. [cited 2020 Oct 13].

46. Caris MG, Labuschagne HA, Dekker M, Kramer MHH, van Agtmael MA, Vandenbroucke-Grauls CMJE. Nudging to improve hand hygiene. J Hosp Infect. 2018;98(4):352-8.

47. Srigley JA, Corace K, Hargadon DP, Yu D, MacDonald T, Fabrigar L, et al. Applying psychological frameworks of behaviour change to improve healthcare worker hand hygiene: a systematic review. J Hosp Infect. 2015; 91(3):202-10.

48. Grieco D, Lacetera N, Macis M, Di Martino D. Motivating cord blood donation with information and behavioral nudges. Sci Rep. 2018;8(1): 252.

49. Yoong SL, Hall A, Stacey F, Grady A, Sutherland R, Wyse R, et al. Nudge strategies to improve healthcare providers' implementation of evidence-based quidelines, policies and practices: a systematic review of trials included within Cochrane systematic reviews. Implement Sci. 2020;15(1):1-30.

50. Beidas RS, Adams DR, Kratz HE, Jackson K, Berkowitz S, Zinny A, et al. Lessons learned while building a trauma-informed public behavioral health system in the City of Philadelphia. Eval Program Plan. 2016;59:21-32.

51. Philadelphia - State of the Child County Profile. Pennsylvania partnership for children; 2017. Available from: https://www.papartnerships.org/wp-content/ uploads/2018/04/Philadelphia-2017-SOTC-Profile.pdf.

52. de Arellano MAR, Lyman DR, Jobe-Shields L, George P, Dougherty RH, Daniels AS, et al. Trauma-focused cognitive-behavioral therapy for children and adolescents: assessing the evidence. Psychiatric Serv. 2014; 65(5):591-602.

53. Mavranezouli I, Megnin-Viggars O, Daly C, Dias S, Stockton S, Meiser-Stedman $R$, et al. Research review: psychological and psychosocial treatments for children and young people with post-traumatic stress disorder: a network meta-analysis. J Child Psychol Psychiatry. 2020;61(1):18-29.

54. Cohen JA, Deblinger E, Mannarino AP. Trauma-focused cognitive behavioral therapy for children and families. Psychother Res. 2018;28(1):47-57. 
55. Amaya-Jackson L, Hagele D, Sideris J, Potter D, Briggs EC, Keen L, et al. Pilot to policy: statewide dissemination and implementation of evidence-based treatment for traumatized youth. BMC Health Serv Res. 2018;18(1):589.

56. Deblinger E, Mannarino AP, Cohen JA, Runyon MK, Steer RA. Traumafocused cognitive behavioral therapy for children: impact of the trauma narrative and treatment length. Depress Anxiety. 2011;28(1):67-75.

57. Stewart RE, Beidas RS, Last BS, et al. Applying NUDGE to Inform Design of EBP Implementation Strategies in Community Mental Health Settings [published online ahead of print, 2020 May 19]. Adm Policy Ment Health. 2020. https://doi.org/10.1007/s10488-020-01052-z.

58. The Behavioral Economics Guide 2018. Behavioral Economics Group; 2018. (Samson A, editor. The Behavioral Economics Guide). Available from: https:// www.behavioraleconomics.com/the-be-guide/the-behavioral-economicsguide-2018/. Accessed 7 Jan 2018.

59. The Behavioural Insights Team. EAST: four simple ways to apply behavioural insights. London, UK; 2014. Available from: https://www.bi.team/wp-content/ uploads/2015/07/BIT-Publication-EAST_FA_WEB.pdf. [cited 2020 Oct 1].

60. Frank HE, Fishman J, Lushin V, Last BS, Jackson K, Berkowitz S, et al. Perceived barriers and facilitators to use of trauma narratives: theory-based belief elicitations. Rockville: Biennial National Institutes of Mental Health Services Research Conference; 2018

61. Lyle J. Stimulated recall: a report on its use in naturalistic research. Br Educ Res J. 2003;29(6):861-78.

62. Braun V, Clarke V, Hayfield N. Thematic analysis. Smith JA, editor. London: Sage Publications; 2015. p. 222-48.

63. Manoogian J, Benson B. Cognitive bias codex. 2017. Available from: https:// www.sog.unc.edu/sites/www.sog.unc.edu/files/course_materials/ Cognitive\%20Biases\%20Codex.pdf. [cited 2020 Nov 25].

64. Hærem T, Pentland BT, Miller KD. Task complexity: extending a core concept. Acad Manage Rev. 2015;40(3):446-60.

65. Sintchenko V, Coiera E. Decision complexity affects the extent and type of decision support use. In: AMIA Annual Symposium Proceedings. Washington, DC: American Medical Informatics Association; 2006. p. 724

66. Johnson EJ, Shu SB, Dellaert BG, Fox C, Goldstein DG, Häubl G, et al. Beyond nudges: tools of a choice architecture. Market Lett. 2012;23(2):487-504.

67. Kim RH, Day SC, Small DS, Snider CK, Rareshide CAL, Patel MS. Variations in influenza vaccination by clinic appointment time and an active choice intervention in the electronic health record to increase influenza vaccination. JAMA Netw Open. 2018;1(5):e181770.

68. Drewes AA. Blending play therapy with cognitive behavioral therapy: evidence-based and other effective treatments and techniques. Hoboken: Wiley; 2009.

69. Goodyear-Brown P. Trauma and play therapy: helping children heal. New York: Routledge; 2019.

70. Cohen K, Collens P. The impact of trauma work on trauma workers: a metasynthesis on vicarious trauma and vicarious posttraumatic growth. Psychol Trauma. 2013;5(6):570.

71. Jenkins SR, Baird S. Secondary traumatic stress and vicarious trauma: a validational study. J Trauma Stress. 2002;15(5):423-32.

72. Loewenstein G. Hot-cold empathy gaps and medical decision making Health Psychol. 2005;24(4S):S49.

73. Smith L, Li V, Dykema S, Hamlet D, Shellman A. "Honoring somebody that society doesn't honor": therapists working in the context of poverty. J Clin Psychol. 2013;69(2):138-51.

74. Staddon JE, Cerutti DT. Operant conditioning. Ann Rev Psychol. 2003;54(1): $115-44$

75. Carleton RN, Mulvogue MK, Thibodeau MA, McCabe RE, Antony MM, Asmundson GJ. Increasingly certain about uncertainty: intolerance of uncertainty across anxiety and depression. J Anxiety Disord. 2012;26(3):468-79.

76. Agren T, Björkstrand J, Fredrikson M. Disruption of human fear reconsolidation using imaginal and in vivo extinction. Behav Brain Res. 2017; 319:9-15.

77. Frank HE, Becker-Haimes EM, Rifkin LS, Norris LA, Ollendick TH, Olino TM, et al. Training with tarantulas: a randomized feasibility and acceptability study using experiential learning to enhance exposure therapy training. J Anxiety Disord. 2020;76:102308.

78. Schultz PW, Nolan JM, Cialdini RB, Goldstein NJ, Griskevicius V. The constructive, destructive, and reconstructive power of social norms. Psychol Sci. 2007;18(5):429-34.
79. Persell SD, Doctor JN, Friedberg MW, Meeker D, Friesema E, Cooper A, et al. Behavioral interventions to reduce inappropriate antibiotic prescribing: a randomized pilot trial. BMC Infect Dis. 2016;16:373.

80. Damschroder LJ. Clarity out of chaos: use of theory in implementation research. Psychiatry Res. 2020;283:112461.

81. Nilsen P. Making sense of implementation theories, models and frameworks. Implement Sci. 2015;10(1):53.

82. Damschroder LJ, Reardon CM, Lowery JC. The Consolidated Framework for Implementation Research (CFIR). In: Handbook on Implementation Science. Cheltenham: Edward Elgar Publishing; 2020

83. Sweller J. Cognitive load theory. In: Psychology of learning and motivation. Academic Press; 2011. p. 37-76. https://doi.org/10.1016/B978-0-12-387691-1. 00002-8.

84. Last BS, Rudd BN, Gregor CA, Kratz HE, Jackson K, Berkowitz S, et al. Sociodemographic characteristics of youth in a trauma focused-cognitive behavioral therapy effectiveness trial in the city of Philadelphia. J Commun Psychol. 2020;48(4):1273-93.

85. Rudd BN, Last BS, Gregor C, Jackson K, Berkowitz S, Zinny A, et al. Benchmarking treatment effectiveness of community-delivered traumafocused cognitive behavioral therapy. Am J Commun Psychol. 2019;64(3-4): 438-50.

86. Bright TJ, Wong A, Dhurjati R, Bristow E, Bastian L, Coeytaux RR, et al. Effect of clinical decision-support systems: a systematic review. Ann Intern Med. 2012;157(1):29-43

87. Cohen J, Mannarino AP. Disseminating and implementing trauma-focused CBT in community settings. Trauma Violence Abuse. 2008;9(4):214-26.

88. Sigel BA, Kramer TL, Conners-Burrow NA, Church JK, Worley KB, Mitrani NA. Statewide dissemination of trauma-focused cognitive-behavioral therapy (TF-CBT). Child Youth Serv Rev. 2013;35(6):1023-9.

89. Sigel BA, Benton AH, Lynch CE, Kramer TL. Characteristics of 17 statewide initiatives to disseminate trauma-focused cognitive-behavioral therapy (TFCBT). Psychol Trauma. 2013;5(4):323.

90. Ebert L, Amaya-Jackson L, Markiewicz JM, Kisiel C, Fairbank JA. Use of the breakthrough series collaborative to support broad and sustained use of evidence-based trauma treatment for children in community practice settings. Adm Policy Ment Health Ment Health Serv Res. 2012;39(3):187-99.

91. Murray LK, Dorsey S, Skavenski S, Kasoma M, Imasiku M, Bolton P, et al. Identification, modification, and implementation of an evidence-based psychotherapy for children in a low-income country: the use of TF-CBT in Zambia. Int J Ment Health Syst. 2013;7(1):24.

92. Webb C, Hayes A, Grasso D, Laurenceau J-P, Deblinger E. Trauma-focused cognitive behavioral therapy for youth: effectiveness in a community setting. Psychol Trauma. 2014;6(5):555-62.

93. Bass J, Bearup L, Bolton P, Murray L, Skavenski S. Implementing trauma focused cognitive behavioral therapy (TF-CBT) among formerly traffickedsexually exploited and sexually abused girls in cambodia: a feasibility study. Phnom Penh, Cambodia: World Vision; 2011.

94. Asch DA, Rosin R. Innovation as discipline, not fad. New England J Med. 2015;373(7):592-4

95. Sedikides C, Gaertner L, Cai H. On the panculturality of self-enhancement and self-protection motivation: the case for the universality of self-esteem. In: Advances in motivation science. New York: Elsevier; 2015. p. 185-241.

96. Skriner LC, Wolk CB, Stewart RE, Adams DR, Rubin RM, Evans AC, et al. Therapist and organizational factors associated with participation in evidence-based practice initiatives in a large urban publicly-funded mental health system. J Behav Health Serv Res. 2018;45(2):174-86.

97. Bruns EJ, Parker EM, Hensley S, Pullmann MD, Benjamin PH, Lyon AR, et al. The role of the outer setting in implementation: associations between state demographic, fiscal, and policy factors and use of evidence-based treatments in mental healthcare. Implement Sci. 2019;14(1):96.

\section{Publisher's Note}

Springer Nature remains neutral with regard to jurisdictional claims in published maps and institutional affiliations. 